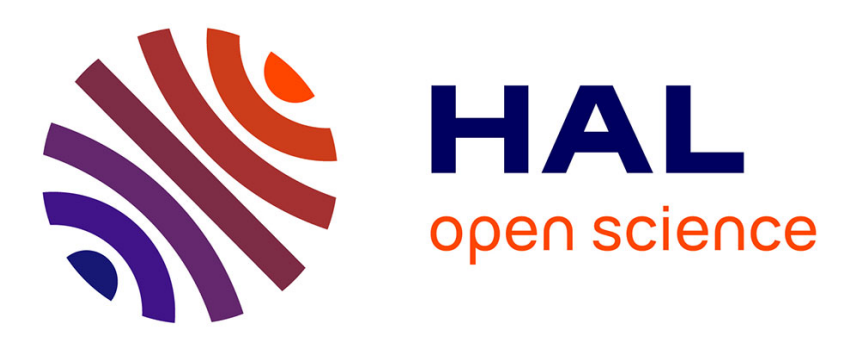

\title{
Contrôlabilité exacte de la population des états propres dans les systèmes quantiques bilinéaires
}

\author{
Gabriel Turinici
}

\section{To cite this version:}

Gabriel Turinici. Contrôlabilité exacte de la population des états propres dans les systèmes quantiques bilinéaires. Comptes rendus de l'Académie des sciences. Série I, Mathématique, 2000, 330 (4), pp.327332. hal-00536515

\section{HAL Id: hal-00536515 https://hal.science/hal-00536515}

Submitted on 8 Mar 2013

HAL is a multi-disciplinary open access archive for the deposit and dissemination of scientific research documents, whether they are published or not. The documents may come from teaching and research institutions in France or abroad, or from public or private research centers.
L'archive ouverte pluridisciplinaire $\mathbf{H A L}$, est destinée au dépôt et à la diffusion de documents scientifiques de niveau recherche, publiés ou non, émanant des établissements d'enseignement et de recherche français ou étrangers, des laboratoires publics ou privés. 


\title{
Contrôlabilité exacte de la population des états propres dans les systèmes quantiques bilinéaires
}

\author{
Gabriel TURINICI
}

Laboratoire ASCI, UPR 9029, bâtiment 506, Université Paris-Sud, 91405 Orsay cedex, France

(Reçu le 23 octobre 1999, accepté le 13 décembre 1999)

\begin{abstract}
Résumé. On présente dans cette Note des résultats de contrôlabilité exacte pour les systèmes quantiques en interaction avec des lasers. Un premier résultat négatif sur des espaces de dimension infinie sert comme point de départ à une analyse en dimension finie. On montre que sous des hypothèses raisonnables du point de vue physique dans de tels systèmes on peut contrôler la population des états propres. On présente une application pour un système à cinq niveaux. (C) 2000 Académie des sciences/Éditions scientifiques et médicales Elsevier SAS
\end{abstract}

\section{Exact controllability for the population of the eigenstates in bilinear quantum systems}

\begin{abstract}
We present in this Note exact controllability results for quantum systems interacting with lasers. A negative result for infinite-dimensional spaces serves as a starting point for a finite-dimensional analysis. We show that under physically reasonable hypothesis in such systems we can control the population of the eigenstates. Applications are given for a five-level system. (C) 2000 Académie des sciences/Éditions scientifiques et médicales Elsevier SAS
\end{abstract}

\section{Abridged English Version}

(The notations for equations point to the French version.)

Controlling chemical reactions at the quantum level has been a long-lasting goal for the Chemists (cf. [2], [3], [6], [8], [10], [11], [14]) from the very beginning of the laser technology. Experiences have shown that designing the laser pulse able to steer the system to the desired target state is a rather difficult task that physical intuition alone cannot accomplish. It is only recently that tools coming from control theory began to give satisfactory results in some particular cases; finding the optimal electric field is now treated by numerical methods and new models are sought for that be also reliable and cheap computationally. A legitimate question arises in this context: what quantum states can be attained using such an external field? Some answers are given below.

Let $\Psi_{0}$ be the initial state of the system, $H_{0}$ its internal Hamiltonian. In the absence of external interactions the system follow dynamics (1). Once laser pulse is introduced, the new dynamics is given by (2), where $\mathcal{B}$ is some dipole moment operator. The goal is to find (if any) a finite energy $\left(\int \varepsilon(t)^{2} \mathrm{~d} t<\infty\right)$ laser pulse $\varepsilon(t)$ able to steer the system from $\Psi_{0}$ to some predefined target $\Psi_{\text {target }}$.

\footnotetext{
Note présentée par Jacques-Louis LiONS.
}

0764-4442/00/03300000 (c) 2000 Académie des sciences/Éditions scientifiques et médicales Elsevier SAS. Tous droits réservés. 


\section{G. Turinici}

Note that after the laser is switched off $(\varepsilon(t)=0, t>T)$ the system instantaneously modifies its state by the formula (1) at best by a phase factor (but conserves the $\mathrm{L}^{2}$ norm of $\Psi$ ).

A first negative controllability result is therefore not really restrictive. In fact using compacity arguments as those in [1] we have proved that the set of attainable states has dense complement on the unit sphere. Given this result we focus on studying the finite-dimensional controllability.

Let us express (2) with respect to a (known) basis $\left\{\Psi_{i}(x) ; i=1, \ldots, n\right\}$ of a finite-dimensional subspace of $\mathrm{L}_{x}^{2}\left(\mathbb{R}^{3 N}\right)$ and let $A$ and $B$ be matrices corresponding to operators $H_{0}$ and $\mathcal{B}$ respectively; then the evolution equations are (3) with the state of the system being $\Psi(t, x)=\sum_{i=1}^{n} c_{i}(t) \Psi_{i}(x)$. Moreover, suppose for simplicity that $\Psi_{i}(x)$ are eigenfunctions of $H_{0}$. Due to the relative phase change during free evolution of the system we will study only the population transfer between eigenstates, and not relative phases (that is only changes in $\left.\left|c_{i}(t)\right|\right)$. We call population distribution for the system (3) any $n$-tuple $d \in \mathbb{R}^{n}$ such that (5) holds.

We associate to the system a non-oriented graph $G=(S, A)$ by $(6)$ where $S$ is the set of states $\Psi_{i}, i=1, \ldots, n$ and the edge set $A$ corresponds to the states coupled by the matrix $B$; decompose this graph into connected components $G_{\alpha}=\left(S_{\alpha}, A_{\alpha}\right), \alpha=1, \ldots, K$. We show that conservation laws for each component give rise to necessary conditions for controllability: if one can steer the system into the population distribution $d$ (i.e. $\left|c_{\varepsilon k}(T)\right|=d_{k} k=1, \ldots, n$ ), then (8) holds.

Let us now eliminate from $G$ all pairs of edges $(i, j),(a, b)$ that correspond to degenerate transitions $\lambda_{i}-\lambda_{j}=\lambda_{a}-\lambda_{b}$ and obtain some other graph $\widetilde{G}$. The main result states that if $\widetilde{G}$ has not more connected components than $G$ (it cannot have less) then the necessary conditions are also sufficient. The theory is constructive and we apply it to some five-level system (cf. [12]) given by (12). Numerical simulations (see (13)) sustain, on this example, the previous theory for transitions from state 1 to state 5 , from state 1 to state 2 using state 4 and finally from states 1 and 2 to 4 .

\section{Introduction}

Défi récent et intrinsèquement multidisciplinaire, le contrôle du mouvement moléculaire par des lasers a été un but pour nombre de chimistes (voir [2],[3], [6], [8], [10], [11], [14]) dès le début du développement des technologies modernes.

Les premières tentatives basées sur l'intuition physique ont généralement échoué face aux phénomènes de dispersion incontrôlable de l'énergie dans les molécules. Récemment des méthodes systématiques, inspirées par la théorie du contrôle optimal, ont commencé à se développer et donner des résultats pertinents dans l'étude de quelques phénomènes simples.

Il s'agit de contrôler les équations qui interviennent dans la dynamique moléculaire. Soit donc un système (isolé pour le moment) dont l'hamiltonien est noté par $H_{0}$. Ce système obéit aux équations de Schrödinger dépendant du temps. Si on note par $\Psi_{0}$ l'état initial du système et par $\Psi(t)$ son état à l'instant $t$ on aura alors :

$$
i \hbar \frac{\partial}{\partial t} \Psi=H_{0} \Psi, \quad \Psi(t=0)=\Psi_{0},\left\|\Psi_{0}\right\|_{\mathrm{L}_{x}^{2}\left(\mathbb{R}^{3 N}\right)}=1 .
$$

En présence d'une interaction extérieure qui, pour nous, sera un champ électrique créé par un laser et modélisé par l'intensité $\varepsilon(t) \in \mathbb{R}$ du champ laser et par un certain opérateur dipolaire $\mathcal{B}$ indépendant du temps, les équations (de la dynamique contrôlée) s'écrivent :

$$
i \hbar \frac{\partial}{\partial t} \Psi_{\varepsilon}=H_{0} \Psi_{\varepsilon}-\varepsilon(t) \mathcal{B} \Psi_{\varepsilon}=H \Psi_{\varepsilon}, \quad \Psi_{\varepsilon}(t=0)=\Psi_{0} .
$$

Le problème devient alors de trouver le temps final $T$ et le champ $\varepsilon(t) \in \mathrm{L}^{2}([0, T])$ qui permet de diriger dans l'intervalle $[0, T]$ le système de l'état initial $\Psi_{0}$ vers l'état cible $\Psi_{\varepsilon}(x, T)=\Psi_{\text {cible }}(x)$. 
Il convient de remarquer qu'il découle des principes d'incertitude qu'on ne saura jamais vérifier et/ou exploiter une contrôlabilité exacte. En effet, même si une procédure quelconque nous donne en sortie exactement le $\Psi_{\text {cible }}(x)$ désiré, l'évolution libre ultérieure du système quantique modifie instantanément cet état (par une phase dépendant du temps si $\Psi_{\text {cible }}(x)$ est une fonction propre de $H_{0}$ et d'après la formule (1) dans le cas général). Notons par ailleurs que $\varepsilon(t) \in \mathbb{R}, \forall t \leq T$ garantit la conservation de la norme $\mathrm{L}_{x}^{2}\left(\mathbb{R}^{3 N}\right)$ du $\Psi_{\varepsilon}(t, x)$.

Dans ce contexte un premier résultat négatif de contrôlabilité exacte n'est donc nullement restrictif. On a en effet montré à partir des résultats de [1] que le complémentaire par rapport à la sphère unité de $\mathrm{L}_{x}^{2}\left(\mathbb{R}^{3 N}\right)$ de l'ensemble des états atteignables : $\left\{\Psi ;\|\Psi\|_{\mathrm{L}_{x}^{2}\left(\mathbb{R}^{3 N}\right)}=\right.$ $1\} \backslash \bigcup_{T>0}\left\{\Psi_{\varepsilon}(T) ; \varepsilon(t) \in \mathrm{L}^{2}([0, T])\right\}$, est partout dense sur la sphère unité de $\mathrm{L}_{x}^{2}\left(\mathbb{R}^{3 N}\right)$. Au vue de ce résultat, l'étude de la contrôlabilité peut porter soit sur la contrôlabilité d'un nombre fini de moments, soit sur la contrôlabilité du système de dimension finie associé. C'est la deuxième voie qu'on a choisi d'explorer ici.

Soit donc $D=\left\{\Psi_{i}(x) ; i=1, \ldots, n\right\}$ une base orthonormée pour un sous-espace de dimension finie de $\mathrm{L}_{x}^{2}\left(\mathbb{R}^{3 N}\right)$ qui nous intéresse ${ }^{1}$ et $A$ respectivement $B$ les matrices des opérateurs $H_{0}$ respectivement $\mathcal{B}$ dans cette base ${ }^{2}$. Si on note par $C=\left(c_{i}\right)_{i=1}^{n}$ les coefficients des $\Psi_{i}(x)$ dans l'expression de la solution $\Psi(t, x)=\sum_{i=1}^{n} c_{i}(t) \Psi_{i}(x)$ alors les équations (2) deviennent

$$
i \hbar \frac{\partial}{\partial t} C_{\varepsilon}=A C_{\varepsilon}-\varepsilon(t) B C_{\varepsilon}, \quad C_{\varepsilon}(t=0)=C_{0}, \quad \text { où } \quad C_{0}=\left(c_{0 i}\right)_{i=1}^{n}, \quad c_{0 i}=\int_{\mathbb{R}^{3 N}} \Psi_{0} \Psi_{i} \mathrm{~d} x .
$$

La contrôlabilité du système (3) a été abordée dans la littérature (voir [9]) en ramenant le problème à la contrôlabilité d'un système sur l'espace des matrices u nitaires de dimension $n$. On a ainsi accès aux résultats généraux sur la contrôlabilité des systèmes bilinéaires sur des groupes de Lie. Cette approche ne correspond néanmoins pas à une nécessité physique, elle donne lieu à des critères assez lourds à vérifier et donne seulement des conditions suffisantes. Finalement, il existe une large classe de systèmes quantiques simples contrôlables (dans un sens qu'on détaillera ensuite) mais qui ne vérifient pas les critères proposés. Il nous a paru donc intéressant d'aborder le problème en tenant compte de la spécificité du cadre quantique ; on a ainsi pu trouver les conditions nécessaires et suffisantes pour la contrôlabilité en dimension finie ${ }^{3}$.

\section{Contrôlabilité en dimension finie-préliminaires}

Dans le cas de la modélisation, la matrice $A$ est diagonale et $B$ est une matrice symétrique réelle à diagonale nulle. Soit $\lambda_{i}, i=1, \ldots, n$, les éléments diagonaux de $A$ (les énergies des états $\left.\Psi_{i}\right)^{4}$.

Avant la présentation des résultats il nous reste à formaliser notre concept de contrôlabilité. Comme on a vu auparavant le système conserve la norme $\mathrm{L}^{2}$ ce qui dans la représentation en dimension finie s'écrit (les $\Psi_{k}$ étant orthonormées) :

$$
\sum_{i=1}^{n}\left|c_{\varepsilon i}(t)\right|^{2}=1, \quad \forall t \geq 0 .
$$

De plus le système libre $((3)$ avec $\varepsilon(t)=0)$ fait varier les phases relatives des états $\Psi_{i}$ mais laisse les populations des états propres (les $\left|c_{i}\right|^{2}$ ) inchangées.

DÉfinition 1. - On appelle distribution de population pour le système (3) un $d \in \mathbb{R}^{n}$ tel que

$$
d=\left(d_{k}\right)_{k=1}^{n}, \quad d_{k} \geq 0, \quad k=1, \ldots, n, \quad \sum_{k=1}^{n} d_{k}^{2}=1 .
$$




\section{Graphe de transfert et conditions nécessaires}

Conformément à l'intuition physique qu'on confortera dans la suite par des arguments mathématiques, la matrice $B$ décrit le transfert de population entre les différents états propres du système. Afin de formaliser cette observation on introduit le graphe de transfert du système $G=(S, A)$ où l'ensemble des sommets est l'ensemble des états propres du système et l'ensemble des arêtes est l'ensemble des paires d'états couplés par $B$. Comme $B$ est symétrique on considère $G$ non-orienté.

$$
G=(S, A): S=\left\{\Psi_{1}, \ldots, \Psi_{n}\right\} \quad A=\left\{\left(\Psi_{i}, \Psi_{j}\right) ; b_{i j} \neq 0\right\} .
$$

On va noter dans la suite $\operatorname{par} G_{\alpha}=\left(S_{\alpha}, A_{\alpha}\right), \alpha=1, \ldots, K$, les composantes connexes de $G$. Quitte à faire des permutations sur les indices cette partition correspond à une structure blocdiagonale de la matrice $B$. On en déduit alors des nouvelles lois de conservation qui s'écrivent:

$$
\sum_{\left\{i ; \Psi_{i} \in S_{\alpha}\right\}}\left|c_{\varepsilon i}(t)\right|^{2}=\text { constant, } \forall t \in[0, T], \forall \alpha=1, \ldots, K
$$

Pour justifier (7) on vérifie par la définition de $G$ et en utilisant les équations (3) que pour tout $\alpha=1, \ldots, K: i \hbar \frac{\partial}{\partial t} \sum_{\left\{i ; \Psi_{i} \in S_{\alpha}\right\}}\left|c_{\varepsilon i}(t)\right|^{2}=0$. Ceci nous permet de donner des conditions nécessaires pour la contrôlabilité :

Lemme 1. - Soit $C_{0}$ une configuration initiale et $d$ une distribution de population (cible). S'il existe $T_{d}$ et $\varepsilon(t) \in \mathrm{L}^{2}\left(\left[0, T_{d}\right]\right)$ tel que $\left|c_{\varepsilon k}\left(T_{d}\right)\right|=d_{k}, k=1, \ldots, n$, alors

$$
\sum_{\left\{i ; \Psi_{i} \in S_{\alpha}\right\}}\left|c_{0 i}\right|^{2}=\sum_{\left\{i ; \Psi_{i} \in S_{\alpha}\right\}} d_{i}^{2}, \quad \forall \alpha=1, \ldots, K .
$$

Définition 2. - On dit que le système (3) est contrôlable en la population si pour tout état $C_{0}$ et toute distribution de population $d$ (sauf éventuellement un ensemble de mesure canonique nulle) qui satisfont la condition nécessaire (8) il existe $T_{d}>0$ et $\varepsilon(t) \in \mathrm{L}^{2}\left(\left[0, T_{d}\right]\right)$ tel que $d=\left\{\left|c_{\varepsilon k}\left(T_{d}\right)\right|\right\}_{k=1}^{n}$.

\section{Conditions suffisantes de contrôlabilité}

Afin de donner le résultat principal de contrôlabilité on introduit l'hypothèse suivante :

H. les composantes $G_{\alpha}, \alpha=1, \ldots, K$, de $G$ restent connexes après élimination des toutes les paires d'arêtes $\left(\Psi_{i}, \Psi_{j}\right),\left(\Psi_{a}, \Psi_{b}\right)$ telles que $\lambda_{i}-\lambda_{j}=\lambda_{a}-\lambda_{b},(i, j) \neq(a, b)$.

ThÉORÈme 1. - Sous l'hypothèse $(\mathrm{H})$ le système (3) est contrôlable en la population.

Démonstration. - Avec le changement de variable $w_{\varepsilon k}(t)=\mathrm{e}^{i \lambda_{k} t} c_{\varepsilon k}(t), k=1, \ldots, n,(3)$ devient :

$$
i \hbar \frac{\partial}{\partial t} w_{\varepsilon k}(t)=\sum_{\ell \neq k} \varepsilon(t) \mathrm{e}^{i\left(\lambda_{k}-\lambda_{\ell}\right) t} b_{k \ell} w_{\varepsilon \ell}(t), \quad k=1, \ldots, n, \quad w_{\varepsilon k}(t=0)=c_{0 k} .
$$

Comme $\left|w_{\varepsilon k}(t)\right|=\left|c_{\varepsilon k}(t)\right|, \forall t \geq 0$, étudier la contrôlabilité de (3) revient à étudier la contrôlabilité de $(9)$. Soit $W_{\varepsilon}(t)=\left(w_{\varepsilon i}(t)\right)_{i=1}^{n}$. Pour alléger l'écriture on supposera qu'on travaille en unités atomiques $(\hbar=1)$. En vue de notre définition de la contrôlabilité on comprend qu'il s'agit en effet de "transférer de la population » entre différents noeuds du graphe $G$ (la quantité totale de population restant constante).

Dans tout ce qui suit pour simplifier la présentation on étudie seulement le cas $G$ connexe $(K=1)$ et $\lambda_{i}-\lambda_{j} \neq \lambda_{a}-\lambda_{b}, \forall(i, j) \neq(a, b)$, le cas général n'apportant pas de concepts nouveaux ${ }^{5}$. 
Définissons un transfert élémentaire de $\mu$ unités de population entre les modes $\Psi_{k}$ et $\Psi_{\ell}$ par :

$$
\left|w_{\varepsilon k}(T)\right|^{2}=\left|w_{\varepsilon k}(0)\right|^{2}-\mu, \quad\left|w_{\varepsilon \ell}(T)\right|^{2}=\left|w_{\varepsilon \ell}(0)\right|^{2}+\mu, \quad\left|w_{\varepsilon i}(T)\right|=\left|w_{\varepsilon i}(0)\right|, \quad \forall i \neq k, \ell ;
$$

alors il est facile à voir que notre problème de " transfert de population 》 peut être décomposé en (au plus) $n-1$ transferts élémentaires de population entre les modes propres.

Il nous reste à montrer que pour chaque transfert tel que $b_{k \ell} \neq 0$ on peut trouver $T_{k \ell}>0$ et une telle transformation dans l'intervalle $\left[0, T_{k \ell}\right]$. Choisissons $\varepsilon(t)=\frac{1}{p} r_{k \ell} \cos \left(\lambda_{k}-\lambda_{\ell}\right) t$. Alors on peut écrire ${ }^{6}$ la solution de (9) sous forme vectorielle :

$$
W_{\varepsilon}(p T) \simeq \mathrm{e}^{-i M T} W_{\varepsilon}(0), \text { où } M=\left(\left(\delta_{a k} \delta_{b \ell}+\delta_{a \ell} \delta_{b k}\right) \frac{b_{k \ell} r_{k \ell}}{2}\right)_{a, b=1}^{n} .
$$

Afin d'obtenir la contrôlabilité exacte on montre un résultat de contrôlabilité locale (en la population) par une analyse faisant intervenir le théorème des fonctions inverses dans le même esprit que [1], p. 579 (voir [13]). On trouve ensuite le coefficient $r_{k \ell}$ qui réalise le transfert exact pour la dynamique donnée par la matrice $M$ puisque la dynamique est maintenant réduite à deux états.

Remarque 1. - L'hypothèse $(\mathrm{H})$ admet une interprétation physique : le système n'a pas de dégénérescence de transitions. D'ailleurs on peut donner très facilement des exemples où dans l'absence de cette hypothèse le système n'est pas contrôlable. Il faut néanmoins noter que dans les cas rencontrés dans la pratique (voir [9], [12]) cette hypothèse (H) est vérifiée ${ }^{7}$.

Remarque 2. - Même si notre démarche est constructive elle n'est pas optimale ; il est en effet facile de voir qu'on peut réduire le temps nécessaire pour arriver à la cible en faisant des transformations élémentaires simultanées ${ }^{8}$. On conjecture néanmoins que pour le type de contrôle utilisé la norme $\mathrm{L}^{2}$ de $\varepsilon(t)$ qui réalise le transfert reste constante.

\section{Application}

Comme application des résultats ci-dessus on étudiera un exemple de système à cinq niveaux (voir [12]). On verra que la contrôlabilité est facilement vérifiable par notre méthode et on profitera du côté constructif de la théorie pour quelques illustrations numériques. Les représentations matricielles des opérateurs et le graphe de transfert correspondant sont respectivement :

$$
A=\left(\begin{array}{ccccc}
1.0 & 0 & 0 & 0 & 0 \\
0 & 1.2 & 0 & 0 & 0 \\
0 & 0 & 1.3 & 0 & 0 \\
0 & 0 & 0 & 2.0 & 0 \\
0 & 0 & 0 & 0 & 2.15
\end{array}\right), B=\left(\begin{array}{lllll}
0 & 0 & 0 & 1 & 1 \\
0 & 0 & 0 & 1 & 1 \\
0 & 0 & 0 & 1 & 1 \\
1 & 1 & 1 & 0 & 0 \\
1 & 1 & 1 & 0 & 0
\end{array}\right), G:
$$

Comme $G$ est évidement connexe et de plus l'hypothèse $(\mathrm{H})$ est vérifiée on en déduit la contrôlabilité en la population. Quelques exemples de transfert de population entre les états propres du système sont donnés ensuite. Dans tous les cas on met en évidence l'évolution des $\left|c_{i}(t)\right|^{2}$, $i=1, \ldots, 5$. Pour alléger l'écriture on va noter le transfert désiré par $\left(\left|c_{i}(0)\right|^{2}\right)_{i=1}^{5} \rightarrow\left(\left|c_{i}(T)\right|^{2}\right)_{i=1}^{5}$. On présente ensuite des résultats pour des transferts de population :

1. direct : de l'état 1 à l'état $5:(1,0,0,0,0) \rightarrow(0,0,0,0,1)$ (trivial) ;

2. indirect : de l'état 1 à l'état 2 par l'état $4:(1,0,0,0,0) \rightarrow(0,1,0,0,0)$ (simple) ; 


\section{G. Turinici}

3. coopératif : des états 1 et 2 vers l'état $4:\left(\frac{1}{3}, \frac{2}{3}, 0,0,0\right) \rightarrow(0,0,0,1,0)$ (intéressant).
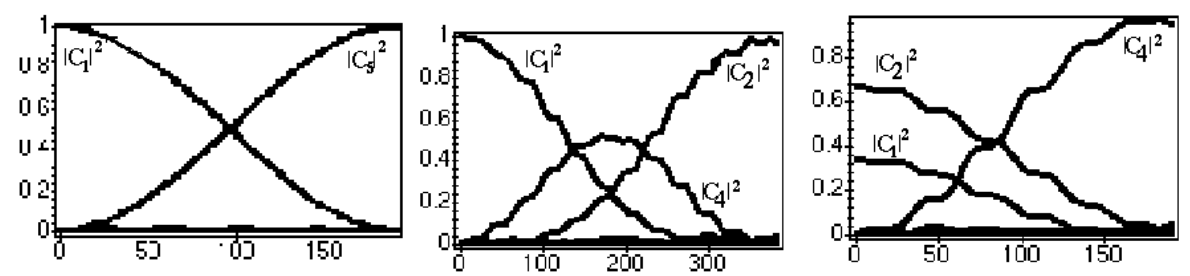

Remarque 3. - La vitesse de transfert est essentiellement déterminée par la matrice $B$ : en regardant le temps nécessaire pour les trois expériences on note que pour des contrôles de même ordre en taille $\mathrm{L}^{\infty}$ la deuxième prend deux fois plus de temps puisque la population doit passer de $\Psi_{1}$ à $\Psi_{4}$ et de $\Psi_{4}$ à $\Psi_{2}$, ce qui peut être réalisé par deux simulations consécutives du premier type.

Remarque 4. - Avec des valeurs en $\mathrm{O}(1)$ dans la matrice $B$ pour avoir une précision de l'ordre $10^{-2}$ notre méthode requiert heuristiquement un $\langle p\rangle$ de l'ordre $\mathrm{O}\left(10^{-2}\right)$ ce qui est consistant avec les temps obtenus et du même ordre que ce qui est obtenu dans la littérature (voir [12]).

\footnotetext{
${ }^{1}$ Cet espace résulte de la modélisation, les fonctions $\Psi_{i}(x)$ étant généralement les premières fonctions propres de $H_{0}$ construites à partir d'un calcul préalable ou encore d'une modélisation basée sur des observations.

${ }^{2}$ On suppose que $\mathcal{B}$ est tel que $b_{i i}=0, i=1, \ldots, n$; pour le cas général voir [13].

3 voir aussi [4], pp. 28-37, pour une présentation de la problématique.

${ }^{4}$ On peut montrer que les conclusions restent vraies avec des adaptations triviales dans le cas d'une base quelconque : par un changement de base on peut rendre en effet $A$ diagonale ; les éléments réels (car $H_{0}$ est hermitien) ainsi obtenus sur la diagonale seront assimilés aux énergies $\lambda_{i}$ employées dans la suite ; pourtant dans ce cas général les $\left|c_{i}\right|^{2}$ n'ont pas le sens classique de population.

5 on décompose le système en plusieurs sous-systèmes qu'on traite indépendamment.

${ }^{6}$ l'approximation héuristiquement en $\mathrm{O}\left(\frac{1}{p}\right)$ devient exacte dans la limite $p \rightarrow \infty$.

7 Dans certaines situations où notre théorie ne s'applique pas il existent des résultats partiels positifs ; c'est le cas de l'oscillateur harmonique (voir [7]). Notons cependant dans [7] le besoin d'un régime perturbatif particulier.

${ }^{8}$ Cette approche peut être rendue rigoureuse par des techniques venant de la théorie des transports distributifs sur des graphes, voir [5] et [13].
}

\section{Références bibliographiques}

[1] Ball J.M., Madersen J.E., Slemrod M., "Controllability for distributed bilinear systems", SIAM J. Control and Optim 20 (4) (1982) 575-597.

[2] Le Bris C., "Control theory applied to quantum chemistry: some tracks", ESAIM PROC (to appear).

[3] Brumer P., Shapiro M., "Coherence Chemistry: Controlling Chemical Reactions with Lasers", Acc. Chem Res. 22, 12 (1989) 407-413.

[4] Butkovskiy A.G., Samoilenko Yu.I., "Control of quantum-mechanical processes and systems", Kluwer, 1990.

[5] Churchmann C.W., Ackoff L., Arnoff E., "Introduction à la recherche opérationnelle", Dunod, Paris, 1970.

[6] Huang G.M., Tarn T.J., Clark J.W., "On the controllability of quantum-mechanical systems", J. Math. Phys. 24, 11 (1983) 2608-2618.

[7] Kime K., "Control of transition probabilities of the quantum-mechanical harmonic oscillator", Appl. Math. Lett. 6 (3) (1993) 11-15.

[8] Kobayashi M., "Mathematics make molecules dance", SIAM NEWS 24 (1998).

[9] Ramakrishna V. et al., "Controlability of molecular systems" Phys. Rev. A 51 (2) (1995) 960-966.

[10] Shi S., Rabitz H., "Optimal control of selectivity of unimolecular reactions via an excited electronic state with designed lasers", Chem. Phys. 97 (1992) 276-287.

[11] Tannor D.J., Rice S.A., "Control of selectivity of chemical reaction via control of wave packet evolution", J. Chem. Phys. 83 (1985) 5013-5018.

[12] Tersigni S.H. et al., "On using shaped light pulses to control the selectivity of product formation in a chemical reaction: An application to a multiple level system", J. Chem. Phys. 93, 3(1990) 1670-1680.

[13] Turinici G., "On the controllability of bilinear quantum systems", in: M. Defranceschi, C. Le Bris (Eds.), Lect. Notes in Chemistry, Springer, (to appear).

[14] Warren W.S., Rabitz H., Dahleh M., "Coherent control of quantum dynamics : the dream is alive", Science 259 (1993) 1581-1589. 